\title{
Erratum to: Biodegradable Polyphosphazene-Based Blends for Regenerative Engineering
}

\author{
Kenneth S. Ogueri ${ }^{1,2,3}$ • Jorge L. Escobar Ivirico ${ }^{2,3,4}$ • Lakshmi S. Nair ${ }^{1,2,3,4,5}$. \\ Harry R. Allcock ${ }^{6}$ - Cato T. Laurencin ${ }^{1,2,3,4,5,7}$
}

Published online: 21 February 2017

(C) The Regenerative Engineering Society 2017

Erratum to: Regen. Eng. Transl. Med.

DOI: 10.1007/s40883-016-0022-7

This paper should have published with the following acknowledgement:

Acknowledgments Support from NIH DP1 AR068147 and the Raymond and Beverly Sackler Center for Biomedical, Biological, Physical and Engineering Sciences, are gratefully acknowledged.

The online version of the original article can be found at http://dx.doi.org/ 10.1007/s40883-016-0022-7

Cato T. Laurencin

Laurencin@uchc.edu

1 Department of Materials Science and Engineering, University of Connecticut, Storrs, CT 06269, USA

2 Institute for Regenerative Engineering, University of Connecticut Health Center, Farmington, CT 06030, USA

3 Raymond and Beverly Sackler Center for Biomedical, Biological, Physical and Engineering Sciences, University of Connecticut Health Center, Farmington, CT 06030, USA

4 Department of Orthopaedic Surgery, University of Connecticut Health Center, Farmington, CT 06030, USA

5 Department of Biomedical Engineering, University of Connecticut, Storrs, CT 06269, USA

6 Department of Chemistry, The Pennsylvania State University, University Park, State College, PA 16802, USA

7 Department of Chemical and Biomolecular Engineering, University of Connecticut, Storrs, CT 06269, USA 